\title{
Review of: "Evaluating Agripreneurs' Satisfaction: Exploring the Effect of Demographics and Emporographics"
}

\author{
Dikky Indrawan ${ }^{1}$ \\ 1 Bogor Agricultural University
}

Potential competing interests: The author(s) declared that no potential competing interests exist.

The research described in this paper may after major revision contribute noteworthy information about Agripreneurs' Satisfaction: Exploring the Effect of Demographics and Emporographics within India for the international peer reviewed literature.

The investigation of the effect of demographics and emporographics on the agripreneurs' satisfaction is important to my knowledge in the context of entrepreneurship. This does add further information that is highly relevant and needs serious consideration by those within the industry and the government agencies responsible for developing agriculture entrepreneurs in India. The substantial effort required by the authors to conduct this research is acknowledged. It has taken time to establish the cooperative relationships needed to conduct this survey.

The authors need to consider the points below and major edits in the manuscript.

The revised manuscript must enable reviewers to fully evaluate the methodology implemented and thus the validity of results presented, and whether the conclusions are justified based on the results.

The revised manuscript must place the work more clearly within the context of published literature sevendimension survey instrument, called AprenSAT, and explain aspects in sufficient detail for international readership to understand entrepreneurship practices in India.

\section{General points to be addressed}

- The Introduction needs to provide an outline of the general agriculture production system in India as the country occupies the leading position in agriculture production and predominantly an agrarian economy

- Please mention India status of agriculture entrepreneurship relative to Asia region or worldwide.

- in production volume to provide context for the reader. As in the third paragraph, the critical sector contributing hugely to the industry and service sector in the form of raw material and other inputs was mentioned with problems but limited information is available on the production system and entrepreneurship in India. Draw on relevant aspects of the production system and entrepreneurship.

- Limited information on problem raised by the authors. A brief description is needed to help reader understand the reasoning to study demographics and emporographics, and satisfaction 
characteristics of agricultural entrepreneurship in India. The third paragraph only mentioned that there is limited information. However, the reason to study different satisfaction characteristics of agricultural entrepreneurship was not build to dissect the problem in India as the leading position. Draw on relevant aspects of recent publication for this and other literature concerning demographics and emporographics. In India

- Methods, need to be more specified.

- Language questionnaire was created in \& process for translation if this was required.

- Sampling methods need further information. So far, limited information is available. Concerning the Salem district in India, the authors should explain. what is the important of Agriculture entrepreneurs in this district compare to the rest of India? Please inform the readers what type of entrepreneurship in Salem the authors to study?

- It seems to use non-probability sampling. Please elaborate, how the sample was selected. Aim of this aspect of the methods is of cause to achieve a representative sample. From my experience conducting research, the research team have encountered common issues re sampling frames etc and taken a generally acceptable, pragmatic approach that ensured inclusion of actors. Further comment to help reader understand the extent to which the proportion of farms finally included in the study is representative of the actual proportion should be mentioned clearly.

- What kind of sampling method used are essential to inform the readers, as the accuracy of statistical methods are impacted by the sampling methods. Further consideration of this as basis for sample size is needed, and if applied, discussion to substantiate its use is required.

- Who were the enumerators? How is the enumerators were selected?

- Ethics and farms visit protocol:

- Do the authors provide ethical clearance or consent for the enumerators?

- Did the enumerators perform specific measures before and during the interview?

- Was there a time period between respondents? Was there a limit to the number of respondents visited per day?

- What was the mean and range for length of time for interview visit?

- Was this by face-to-face interview?

- State clearly the criteria for person interviewed

- Other methods used. In the results sections, some findings or statements came from observations. These findings should be connected to the methods used, for instance observations or else. If the findings came from the questionnaire, then specific information about data collection and data processing should be specified.

- Statistical analysis.

- Please explain and restructure the methods used to prove the hypotheses. Please write in the order of your hypotheses more clearly for each context of the study, so the reader could follow your intention. 


\section{- Results}

- Ensure Titles of Tables provide a full and clear description of content that can be understood by reader without reference to the manuscript text.

- Improve presentation in general of all tables and sub headings. Please consults with the editors.

- Was there any missing data?

- In the results: What is the important factor, what is the important practice?

- Please write the results in a structure that could be followed by readers.

\section{- Discussions}

- Discussion is short of references and the discussion of your findings in relation to similar work in the field. This part is important and interesting. Must include a paragraph that adequately reflects on the strengths and limitations of the methodology applied in the survey and the potential impact of limitations on the validity of results.

- Please provides a good introduction for discussion. The characteristics discussion is limited interconnection with the intended study: the Agripreneurs' Satisfaction level and a positive relationship between Agripreneurs' Satisfaction: Exploring the Effect of Demographics and Emporographics. How can you extrapolate your finding in Salem to the general concept for wider purpose?

- Despite of interesting study, most of the discussion are in general and difficult to follow. The authors could structure the discussion in better way. Please revise it.

- Specific points to be addressed. Note this is not totally comprehensive and it is expected that authors will identify and edit minor points not listed here in the revision to manuscript following to the general point revision 